\title{
Variables pre-analíticas en los análisis de gasometría sanguínea en una institución hospitalaria de tercer nivel, Cali-Colombia
}

\author{
Pre-analytical variables in blood arterial analysis in a third-level hospital institution in Cali, Colombia
}

Johana Sirley Mesa' ${ }^{1}$ orcid.org/0000-0001-9561-9364

Silvana Belalcázar1 orcid.org/0000-0002-1521-1308

Mercedes Salcedo-Cifuentes ${ }^{2^{*}}$ orcid.org/0000-0002-9681-6893

1 Hospital Departamental del Valle del Cauca. Cali, Colombia

2 Grupo de investigación CALIMET, Facultad de Salud, Universidad del Valle. Cali, Colombia

\section{Resumen}

Introducción: Varios factores pre-analíticos afectan los resultados de gases sanguíneos. Objetivo: Evaluar la calidad del proceso pre-analítico de los análisis de gasometría sanguínea solicitados en las unidades de cuidados críticos e intermedios de una institución hospitalaria de tercer nivel de la ciudad de Santiago de Cali. Materiales y métodos: Estudio analítico de corte transversal. Se evaluaron las solicitudes y muestras tomadas a pacientes hospitalizados en las unidades de cuidados críticos. Se calculó medidas de tendencia central, desviación estándar y distribución de proporciones. Los test de Medianas y Kruskal-Wallis permitieron evaluar diferencias estadísticamente significativas en el manejo de la muestra por unidad de servicio/turno/mes/semana de estudio. El análisis se realizó en STATA 15 ${ }^{\circledR}$. Resultados: La muestra efectiva fue de 414 tomadas a 125 pacientes. En promedio se tomaron 4 muestras/día para estudios de gasometría. El $100 \%$ de las solicitudes y muestras no cumplieron con los criterios de calidad y seguridad para la identificación del paciente y $3 \%$ cumplieron con los criterios técnicos de temperatura y tiempos. Conclusiones: Las directrices del Ministerio de Salud para la seguridad del paciente y las normas técnicas de calidad pre-analítica en gasometría no se cumplen.

Palabras clave: Análisis de los gases de sangre; determinación; criterios; métodos de análisis. (Fuente: DeCS, Bireme).

\begin{abstract}
Introduction: Several pre-analytical factors affect the results of blood gases. Objective: To evaluate the quality of the pre-analytical process of the blood arterial analyses requested in the critical and intermediate care units of a thirdlevel hospital institution in the city of Santiago de Cali, Colombia. Materials and methods: A transverse analytical study was conducted. Applications and samples taken to hospitalized patients in critical care units were assessed. Measures of central tendency, standard deviation and distribution of proportions were calculated. The medians and Kruskal-Wallis tests allowed to evaluate statistically significant differences in the management of the sample per unit of service, shift, month, week of study. The analysis was performed in Stata $15^{\circledR}$. Results: The effective sample was 414, which were taken to 125 patients. On average, 4 samples a day were taken for arterial studies. $100 \%$ of applications and samples did not meet the quality and safety criteria for patient identification and $3 \%$ met the technical criteria of temperature and time. Conclusions: The guidelines of the Ministry of Health for patient safety and the technical standards of pre-analytical quality in arterial are not fulfilled.
\end{abstract}

Key words: Blood gas analysis; determination; standards; analytical methods. (Source: DeCS, Bireme). 


\section{Introducción}

El estudio de gasometría sanguínea, es el conjunto de determinaciones analíticas que permiten obtener información rápida y precisa sobre el adecuado funcionamiento del sistema respiratorio y el estado ácido-base de la sangre a través de la medición de $\mathrm{pH}$, presión parcial de oxígeno $\mathrm{PO}_{2}$, presión parcial de dióxido de carbono $\mathrm{PCO}_{2}$ y concentraciones de iones de bicarbonato $[\mathrm{HCO}+3]$ e hidrógeno $\left[\mathrm{H}^{+}\right]^{(1,2)}$.

En el desarrollo de esta prueba clínica intervienen varias fases, la pre-analítica, analítica y postanalítica, cada una de las cuales presentan factores que afectan la calidad de los resultados. En la fase pre-analítica está la mayor probabilidad de introducir errores en la prueba, sin embargo, es la menos estudiada(2). En ella, la trazabilidad en los datos de identificación del paciente( ${ }^{(3)}$, la calidad de las jeringas( ${ }^{(4)}$, el tipo de anticoagulante usado(5), la temperatura de transporte de la muestra (agua helada a $4^{\circ} \mathrm{C}$ )(6), temperatura del paciente ${ }^{(7)}$ y el tiempo de almacenamiento entre la toma de la muestra y su procesamiento(6) son entre otros, factores determinantes en la calidad de los resultados; de ahí la importancia de su estandarización y control permanente.

Un error en la identificación de las muestras biológicas representa un problema grave, con repercusiones directas e inmediatas en la seguridad del paciente(3,4). Según varios estudios internacionales, la proporción de estos errores es significante ${ }^{(6,8)}$. Por otra parte, en la mayoría de los hospitales, las muestras de sangre se toman mediante el uso de jeringas de plástico de polipropileno de alta densidad (impermeables a los gases)(7). El equipo de extracción de sangre adecuado para los estudios de gases sanguíneos es la jeringa plástica heparinizada(8). Sin embargo, se ve el uso de jeringas plásticas no heparinizadas a las que le adicionan de manera manual la heparina lo cual, muy frecuentemente, conlleva a errores en el balance del anticoagulante vs muestra, y con ello a errores en las mediciones llevadas a cabo en gasometría(9-11).

Las condiciones del paciente también afectan los resultados de este análisis clínico. Los equipos miden generalmente el $\mathrm{pH}$, la $\mathrm{PO}_{2}$ y el $\mathrm{PCO}_{2}$ a $37^{\circ} \mathrm{C}$. Por lo tanto, los pacientes con temperatura corporal de $37^{\circ} \mathrm{C}$ o cercana a esta, tendrán resultados exactos que corresponden a los intervalos de referencia, mientras que los pacientes febriles o extremadamente hipotérmicos pueden tener resultados que difieren marcadamente de la verdadera concentración in vivo(7,12).

El tiempo de almacenamiento entre la toma de la muestra y su procesamiento, es otro factor determinante de la calidad de los resultados de gases en sangre. Las células no están ausentes en esta muestra y estas aún mantienen su metabolismo a expensas del consumo de oxígeno, por lo cual la presión de oxígeno puede reportarse baja, asociado a un incremento de la acidificación de la sangre es decir, disminución del $\mathrm{pH}$ relacionado al incremento del lactato(12-14). A este efecto final se le suma la capacidad de los gases de difundir a través de la pared de polipropileno de la jeringa, variable que a su vez está influenciada por la temperatura del medio externo y la cantidad de la muestra(14,15).

Son varios los factores que afectan la calidad de los resultados de los estudios de gasometría, un análisis fundamental en el manejo del paciente crítico. Sin embargo, no se encontró evidencia científica relacionada con el tema en Colombia de aquí que el objetivo del estudio fue evaluar la calidad del proceso pre-analítico de los análisis de gasometría sanguínea solicitados en las unidades de cuidados críticos e intermedios de una institución hospitalaria de tercer nivel de la ciudad de Santiago de Cali.

\section{Materiales y métodos}

Se trató de un estudio analítico de corte transversal llevado a cabo en una institución hospitalaria de tercer nivel de la ciudad de Santiago de Cali.

La población de estudio fue conformada por 1.692 solicitudes y muestras para estudio de gases sanguíneos de pacientes hospitalizados en los servicios de las Unidades de Cuidados Intensivos (UCI) e Intermedio (UCIN) de la institución fuente 
de información, tomadas durante los cuatro meses (diciembre a marzo) que duro el estudio.

Para el cálculo de tamaño de la muestra se consideró la fórmula (16):

$\mathrm{n}=\frac{\mathrm{n}_{\mathrm{o}}}{1+\frac{\mathrm{n}_{\mathrm{o}}}{\mathrm{N}}}$ donde: $\mathrm{n}_{\mathrm{o}}=\mathrm{p}^{*}(1-\mathrm{p})^{*}\left(\frac{z\left(1-\frac{\alpha}{2}\right.}{d}\right)^{2}$

Donde " $n$ " correspondió al tamaño de la población a estudio, este dato se proyectó con base en las estadísticas del 2016 (1.692); "p" fue la proporción de unidades de análisis que cumplían con las especificaciones de calidad pre-analítica para muestras de gasometría sanguínea. Dado que este dato se desconocía, para el momento del diseño del protocolo se consideró tomar el valor de 0,5, como opción más segura. En este sentido, la proporción de casos que no cumplieron con los criterios de calidad pre-analítica en gasometría fue de $1-p=0,5$. Finalmente, el error muestral (d) fue de $5 \%$.Esto conllevó a una muestra de 313 a la cual considerando un porcentaje de pérdida de datos del 30\%, se le sumaron 101 unidades más para una muestra efectiva final de 414.

Se incluyó en el estudio aquellas solicitudes con sus respectivas muestras que llegaron durante el periodo de estudio, procedentes de las unidades de cuidados intensivos (UCI) 2, 3 y 4 y de la unidad de cuidados intermedios (UCIN).

La selección de las unidades de análisis se realizó a través de muestreo aleatorio estratificado. Los estratos fueron los turnos de la mañana, tarde y noche. Esto significó un aporte del 15\% (62/414) tanto para el turno de la mañana como el de la tarde y del 70\% (290/414) para el turno de la noche seleccionados al azar a partir de las UCI2, UCI3, UCI4 y UCIN.

La Tabla 1 presenta un resumen de las variables consideradas en el estudio. Estas se midieron como variables dicotómicas (presente o ausente).

Tabla 1. Grupo de variables consideradas en el estudio

\begin{tabular}{|c|c|}
\hline Variable & Concepto \\
\hline Diagnóstico clínico & $\begin{array}{l}\text { Procedimiento por el cual se identifica una enfermedad o estado de salud de una } \\
\text { persona }\end{array}$ \\
\hline Nombre completo del paciente & Nombre y apellidos completos de acuerdo a documento de identidad presentado \\
\hline No de historia clínica & Correspondiente al número de cedula del paciente \\
\hline Edad & Años cumplidos al momento de la solicitud y toma de muestra \\
\hline Sala de hospitalización & $\begin{array}{l}\text { Dependencia asistencial de la institución donde se encontraba hospitalizado el } \\
\text { paciente: UCI2, UCI3, UCI4 y UCIN }\end{array}$ \\
\hline No de cama & Cama asignada en el servicio de hospitalización \\
\hline Fecha de toma de muestra & Año, mes y día que fue tomada la muestra \\
\hline Hora de toma de muestra & Hora en formato militar a la cual se le toma la muestra \\
\hline Tipo de jeringa usada & Sistema de extracción usado para la toma de muestra \\
\hline Tipo de anticoagulante usado & $\begin{array}{l}\text { Anticoagulante presente en el sistema de extracción o adicionado a la jeringa usada } \\
\text { para la extracción de la muestra }\end{array}$ \\
\hline $\begin{array}{l}\mathrm{T}^{\circ} \text { del paciente al momento de la toma } \\
\text { de la muestra }\end{array}$ & $\begin{array}{l}\text { Medida relativa de calor o frío asociado al metabolismo del cuerpo humano tomada } \\
\text { en grados centígrados }\left({ }^{\circ} \mathrm{C}\right)\end{array}$ \\
\hline $\begin{array}{l}\text { Nombre del médico que ordena el } \\
\text { análisis }\end{array}$ & Nombre y apellido completo del médico tratante \\
\hline $\begin{array}{l}\text { Registro del médico que ordena el } \\
\text { análisis }\end{array}$ & $\begin{array}{l}\text { Es la inscripción, en el sistema de información definido por el Ministerio de Salud } \\
\text { y Protección Social, del talento humano en salud que cumple con los requisitos } \\
\text { establecidos en la Ley } 1164 \text { de } 2007\end{array}$ \\
\hline $\begin{array}{l}\text { Nombre o iniciales de la persona que } \\
\text { toma la muestra }\end{array}$ & Nombre y apellido completo de la persona que tomó la mustra \\
\hline
\end{tabular}


$\mathrm{T}^{\circ}$ de traslado pre-analítico $\left(\mathrm{T}^{\circ} \mathrm{TP}\right) \quad$ Medida relativa de calor o frío asociado al medio de transporte de la muestra tomado en grados centígrados $\left({ }^{\circ} \mathrm{C}\right)$. Fue medida por la diferencia entre varias temperaturas parciales:

-Temperatura del medio de transporte al momento de recibir la muestra en el laboratorio y la T del medio de transporte al momento de su entrega a mensajería en el servicio donde se originó

-Temperatura del medio de transporte de la muestra al momento de su procesamiento en el laboratorio $\mathrm{y}^{\circ}{ }^{\circ}$ del medio de transporte al momento de recibir la muestra en el laboratorio

Tiempo pre-analítico total (TPT) Lapso de minutos que demoró el traslado de la muestra (mx) desde el servicio donde se tomó hasta el laboratorio. Fue medido por la diferencia entre diferentes tiempos parciales:

-Hora de recepción de la muestra en el laboratorio y hora de toma de muestra (mx). -Hora de procesamiento de la muestra $(\mathrm{mx})$ y hora de recepción de la muestra en el laboratorio.

A partir de estas dos variables se calculó: diferencia de tiempo entre entrega de muestra $(\mathrm{mx}) /$ solicitud a mensajería vs toma de muestra (mx); diferencia de tiempo entre recepción de la muestra $(\mathrm{mx})$ /solicitud en el laboratorio vs tiempo de entrega por mensajería y diferencia de tiempo entre procesamiento de la muestra (mx) y recepción de esta con su solicitud en el laboratorio clínico.

$\mathrm{T}^{\circ}=$ temperatura. $\mathrm{T}^{\circ} \mathrm{TP}=$ Temperatura de traslado pre-analítico. $\mathrm{TPT}=$ Tiempo pre-analítico total

\section{Instrumento y recolección de datos}

En una matriz se consolidaron las variables de servicio de origen de la solicitud/muestra y el diagnóstico clínico, así como las especificaciones de calidad de las solicitudes y muestras a evaluar las cuales se apoyaron en las directrices de la Guía de Buenas Prácticas para la Seguridad del Paciente en la Atención en Salud(17). Y en las de la Sociedad Española de Bioquímica y Patología Molecular(18). La medición de las diferentes temperaturas parciales pre-analíticas de la muestra se realizó con un termómetro digital LACOR debidamente calibrado en el rango de $-7^{\circ} \mathrm{C}$ a $25^{\circ} \mathrm{C}$. La medición de los tiempos parciales preanalíticos se llevó a cabo con un cronómetro digital marca CITIZEN. Toda la información fue recolectada por las investigadoras y cuatro asistentes de campo, previamente entrenados.

\section{Análisis de los datos}

A las variables cuantitativas se les calculó sus medidas de tendencia central (media, moda y mediana) y dispersión (DE) y a las variables cualitativas, proporciones y razones. Se valoró la distribución de los datos cuantitativos a través del Sktest. El análisis bivariado se llevó a cabo a través de tabulaciones cruzadas (Tabla de 2 x K) y usando el test de medianas y el Test de Kruskal Wallis (Test KW); de acuerdo a la distribución de los datos, se evaluó si existían diferencias estadísticamente significativas en el manejo de la muestra por unidad de servicio, turno, mes, semana de estudio y día de la semana.

Un análisis de correlación fue hecho entre la temperatura pre-analítica total $\left(\mathrm{T}^{\circ} \mathrm{PT}\right)$ y el tiempo pre-analítico total (TPT) usando el índice de correlación de Pearson. Todos los análisis, las gráficas y las tablas se llevaron a cabo en el software estadístico STATA 15 en su versión modem.

\section{Consideraciones éticas}

Esta investigación fue clasificada como un estudio sin riesgo según Resolución 8430 de 1993 del Ministerio de Salud de Colombia, siguió los lineamientos éticos expuestos en la Declaración de Helsinki de 1964, última revisión en 2002 y fue aprobado por el Comité Institucional de Revisión de Ética de la Universidad del Valle y el de la institución hospitalaria fuente de información. La propuesta fue presentada ante el coordinador de las unidades de cuidados críticos, personal de enfermería y médicos asistenciales de estos servicios quienes apoyaron la recolección de los datos a partir de registros hospitalarios, respetando la confidencialidad de los datos personales de los de los pacientes a quienes se les ordenaron los estudios de gasometría. 


\section{Resultados}

Del total de 414 muestras biológicas con sus respectivas solicitudes procedentes de 125 pacientes que fueron incluidas en el estudio: 172 se recibieron en diciembre, 136 en enero, 34 en febrero y 72 en el mes de marzo. Los días martes, jueves y domingo se solicitó el mayor número de análisis gasométricos $(222 / 414)$. En promedio llegaron 4 muestras por día, con un máximo de 7 , lo cual ocurrió los días domingos. La mayoría de dichas solicitudes se presentaron en las horas de la noche, entre las 23:00 y las 24:00. Los pacientes a quienes se les tomaron las muestras se encontraban hospitalizados en estas unidades por problemas de insuficiencia respiratoria (35\%), encefalopatía tóxica (33\%), sepsis con insuficiencia renal (30\%), y otras patologías (2\%). La Tabla 2 resume los hallazgos de las variables de calidad de la solicitud y la muestra.

Tabla 2. Distribución de los datos mínimos requeridos en la solicitud y la muestra

\begin{tabular}{|c|c|c|}
\hline \multicolumn{3}{|c|}{ Solicitud } \\
\hline & Presente \% & Ausente \% \\
\hline Nombre completo del paciente & & 100 \\
\hline No de historia clínica & 100 & \\
\hline Edad & & 100 \\
\hline Sala de hospitalización & 100 & \\
\hline No de cama & & 100 \\
\hline Hora de toma de muestra & & 100 \\
\hline Tipo de jeringa usada & & 100 \\
\hline Tipo de anticoagulante usado & & 100 \\
\hline $\mathrm{T}^{\circ}$ del paciente al momento de la toma de la muestra & 40 & 60 \\
\hline Nombre del médico que ordena el análisis & & 100 \\
\hline Registro del médico que ordena el análisis & & 100 \\
\hline Nombre de la persona que toma la muestra & & 100 \\
\hline \multicolumn{3}{|c|}{ Muestra } \\
\hline & Presente \% & Ausente \% \\
\hline Nombre completo & & 100 \\
\hline No de historia clínica & 100 & \\
\hline Fecha de toma de muestra & & 100 \\
\hline Hora de toma de muestra & 100 & \\
\hline Sala de hospitalización & & 100 \\
\hline \multicolumn{3}{|l|}{ Numero de cama } \\
\hline Nombre o iniciales de la persona que toma la muestra & & 100 \\
\hline $\mathrm{T}^{\circ}$ del medio de transporte al momento de envío al laboratorio & & 100 \\
\hline
\end{tabular}

En las solicitudes no se hizo referencia al tipo de jeringa utilizada o anticoagulante cuando se usó jeringa no heparinizada. Sin embargo, el grupo de investigadores identificó esta proporción de muestras (9\%), en ella no fue posible identificar el tipo de jeringa y heparina usada. La variable tiempo pre-analítico total (TPT) de la muestra, no presentó una distribución normal (Sktest, $\mathrm{p}<0,05)$. Cuando las muestras fueron trasladadas directamente por el personal de enfermería, la diferencia de tiempo entre la recepción de la muestra en el laboratorio clínico y su toma en el servicio estuvo por debajo de 30 minutos. Los tiempos parciales se resumen en la Figura 1. 


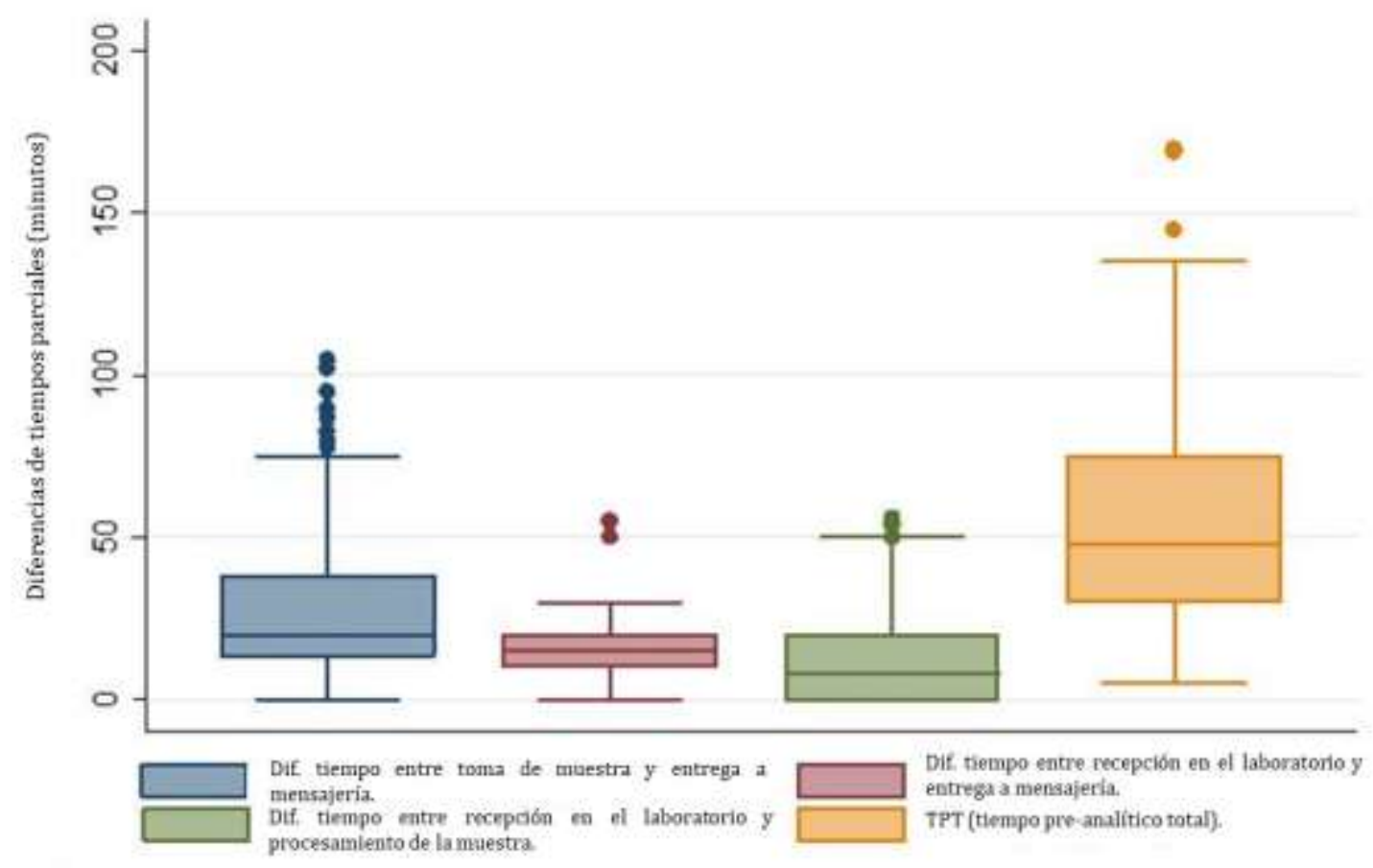

Figura 1. Diferencia de tiempos parciales pre-analíticos de las muestras incluidas en el estudio

La mediana y rangos de las diferencias de los tiempos pre-analíticos parciales considerados en este estudio se presentan en la Tabla 3.

El test de Kruskal-Wallis mostró que las diferencias entre los tiempos pre-analíticos parciales por semana de estudio presentaban diferencias estadísticamente significativas $\mathrm{X}^{2}=$ 71,540; p=0,0001) (Figura 2).

Al evaluar cuantas muestras se manejaron en cada servicio durante el periodo en estudio, se encontró que la UCI2 entregó 129 muestras y la
UCI3 124, estos dos servicios aportaron el 61,1\% de las muestras en estudio.

La temperatura de almacenamiento de las muestras en el medio de transporte al momento de la entrega a mensajería estuvo entre $3,3^{\circ} \mathrm{C}$ a $21,2^{\circ} \mathrm{C}$ y al ser recibidas en el laboratorio entre $3,7^{\circ} \mathrm{C}$ a $21,1^{\circ} \mathrm{C}$. La Figura 3 presenta la distribución de esta variable por turno.

Tomando como referente el TPT de 30 minutos (18)discriminado por servicios, se observó que las muestras procedentes de las UCI 2 y 3 presentaron los datos más prolongados

Tabla 3. Distribución de las diferencias de los tiempos pre-analíticos parciales y el tiempo pre-analítico total de las muestras incluidas en el estudio en minutos

\begin{tabular}{lrr}
\hline Tiempos pre-analíticos parciales & Mediana & \multicolumn{1}{c}{ Rango } \\
\hline Hora de entrega de muestra a mensajería/hora de toma de muestra & 20 & $0-105$ \\
Hora de recepción de la muestra en el laboratorio/hora de entrega de muestra a & 15 & $0-55$ \\
mensajería & 18 & $0-56$ \\
Hora de procesamiento de la muestra en el laboratorio/hora de recepción en el & & $5-170$ \\
laboratorio & 48 & \\
Tiempo pre-analítico total (TPT)
\end{tabular}




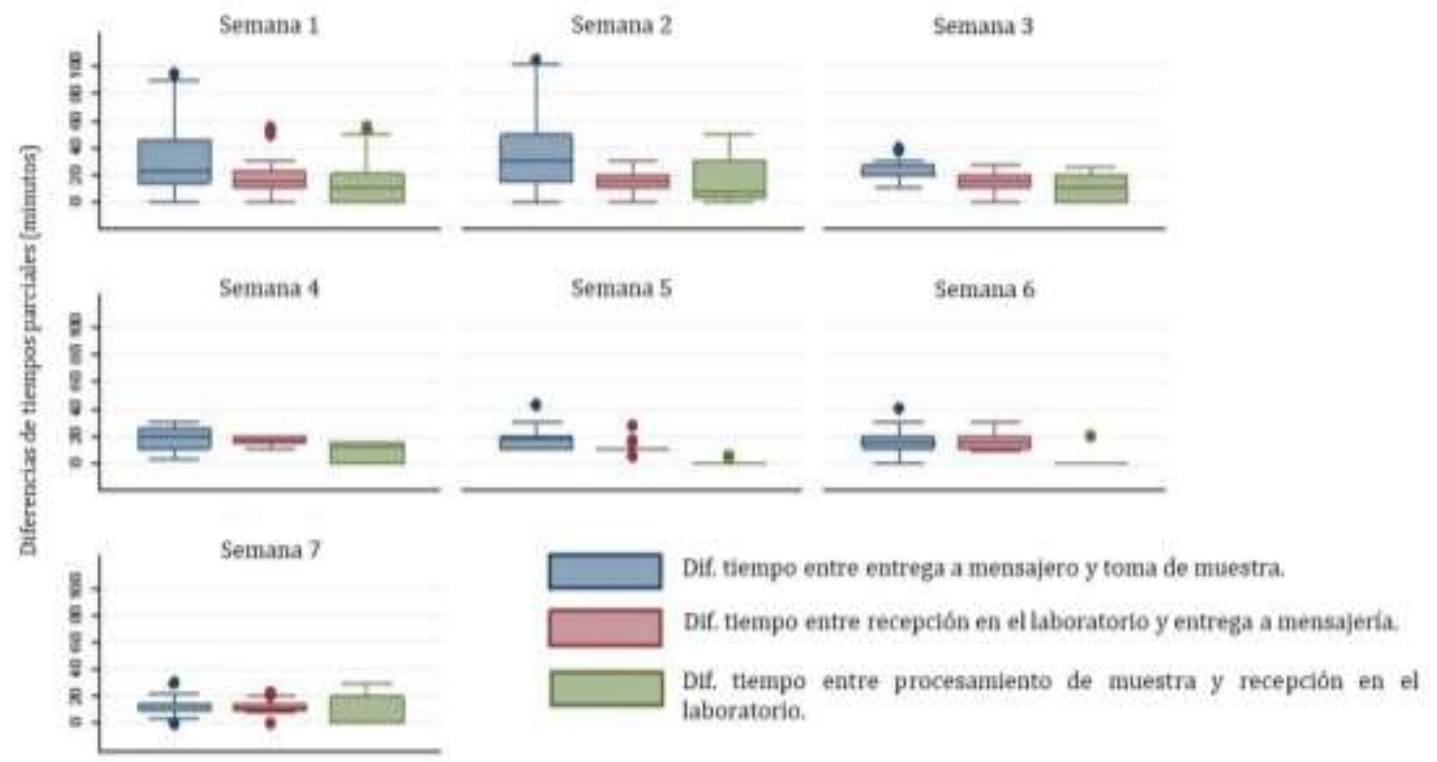

Figura 2. Distribución de los tiempos promedios de manejo pre-analítico de las muestras discriminadas por semana de estudio

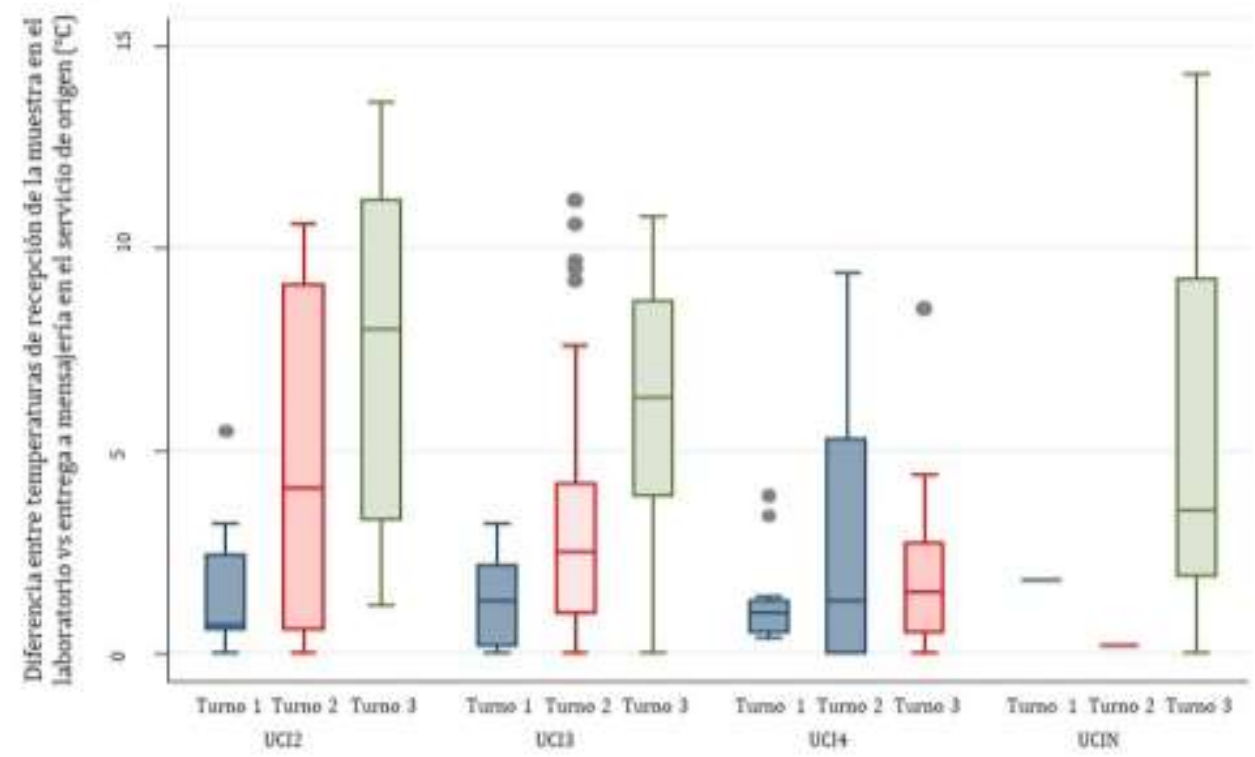

Figura 3. Diferencias de temperaturas $\left({ }^{\circ} \mathrm{C}\right)$ por servicio donde se originó la solicitud con su muestra discriminada por turno

por turno, por encima de 30 minutos, con diferencias estadísticamente significativas en relación con los otros dos servicios, UCI4 y UCIN
(Test KW, $\mathrm{X}^{2}=11,9083 ; \mathrm{p}=0,008$ ). El servicio con menos dispersión en esta variable fue la Unidad de Cuidados Intermedios (Figura 4). 


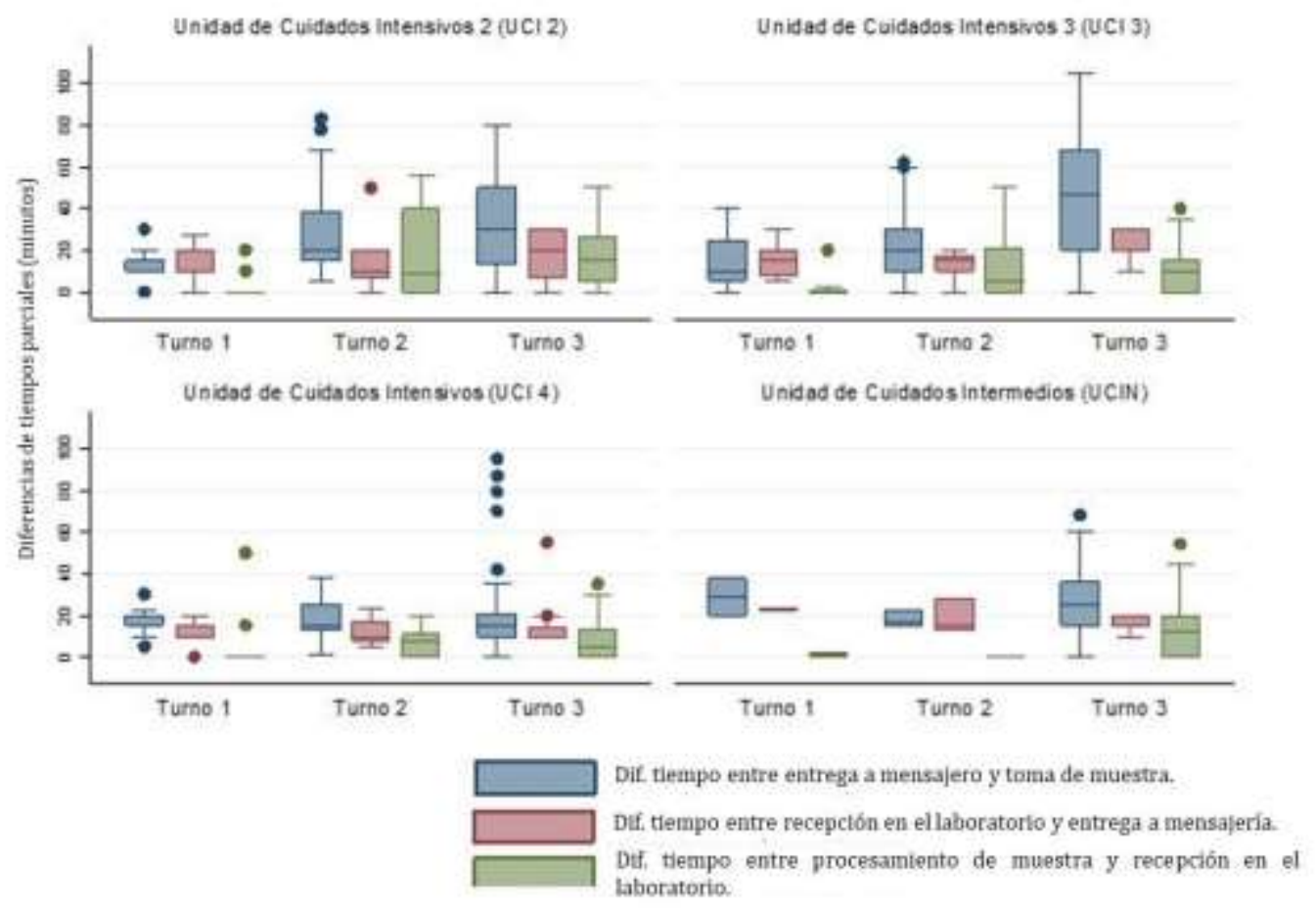

Figura 4. Distribución de los tiempos considerados en el estudio por servicio por turno y servicio

En una tabulación cruzada entre temperatura de transporte óptima $\left(\leq 4^{\circ} \mathrm{C}\right)$ y tiempo de traslado óptimo ( $\leq 30$ minutos), se evidenció que 14 muestras de las 414 cumplieron con los valores establecidos por la Sociedad Española de Bioquímica y Patología Molecular(18), 104/414 muestras llegaron dentro de los primeros 30 minutos pero con temperaturas superiores a $4^{\circ} \mathrm{C}$, 8/414 llegaron con los $4^{\circ} \mathrm{C}$ pero más allá de los 30 minutos y 288/414 llegaron más allá de los 30 minutos con más de $4^{\circ} \mathrm{C}$ (Test de Medianas, $\mathrm{p}=0,047$ ) (Tabla 4).

Para finalizar, se evaluó la correlación entre las variables TPT y $\mathrm{T}^{\circ} \mathrm{PT}$ a través del índice de correlación de Pearson; los resultados mostraron una correlación positiva estadísticamente significante entre las dos variables $(R=0,709$; $\mathrm{p}=0,0001$ ).

Tabla 4. Distribución de las muestras por unidad de cuidado que cumplieron con el estándar de temperatura de almacenamiento pre-analítico y tiempo de traslado

\begin{tabular}{|c|c|c|c|c|c|}
\hline Servicio & $\leq 4^{\circ} \mathrm{C}$ & $>4^{\circ} \mathrm{C}$ & $\begin{array}{l}\text { Tiempo traslado } \\
\leq \mathbf{3 0} \text { minutos }\end{array}$ & $\begin{array}{l}\text { Tiempo traslado } \\
\text { >30 minutos }\end{array}$ & Total de muestras \\
\hline UCI2 & 0 & 129 & 31 & 98 & 129 \\
\hline UCI3 & 4 & 120 & 30 & 94 & 124 \\
\hline UCI4 & 6 & 88 & 49 & 45 & 94 \\
\hline UCIN & 6 & 59 & 9 & 56 & 65 \\
\hline Total de muestras & 16 & 396 & 119 & 293 & 412 \\
\hline
\end{tabular}




\section{Discusión}

Los resultados del estudio mostraron una baja adherencia a directrices o recomendaciones nacionales e internacionales en el manejo adecuado de muestras, específicamente la de gasometría, que pudieran afectar la seguridad del paciente y la toma de decisiones de los médicos en el manejo del paciente crítico.

Las muestras del estudio procedían de pacientes hospitalizados en unidades de cuidados críticos e intermedio, por lo tanto, eran pacientes que presentaban marcadas condiciones de vulnerabilidad clínica, en donde los cambios en $\mathrm{PO}_{2}$ y $\mathrm{PCO}_{2}$ y otros parámetros bioquímicos han mostrado ser determinantes en su pronóstico(19).

Si bien los procedimientos pre-analíticos en este tipo de pruebas, son comunes a todos los exámenes de laboratorio, tienen asociadas ciertas variables que afectan la calidad de los resultados, las cuales están directamente relacionadas con las propiedades físico-químicas de los analitos que se están midiendo. El trabajo evaluó 5 de las 12 variables reportadas en la literatura internacional(20).

Durante el desarrollo del proyecto se evidenció la ausencia de un protocolo institucional para la elaboración de una solicitud médica que cumpla con requisitos mínimos de identificación de los pacientes definidos por el Ministerio de Salud(17) y con datos de relevancia para la toma de decisiones en el laboratorio clínico(18). Según Baird(11), la correcta identificación de la solicitud, la muestra y la concordancia entre los datos registrados en estas dos fuentes, es muy importante para la seguridad del paciente, dado que la toma de decisiones del médico con relación al balance hidroelectrolítico de un paciente crítico, en la mayoría de los casos involucra un resultado de gases en sangre. El rótulo de una muestra para estudio gasométrico debe contener no sólo identificadores únicos del paciente sino también la fecha y hora de toma de la muestra, su ubicación para garantizar que el paciente correcto se esté sometiendo a prueba y que se pueda llamar por teléfono ante un resultado crítico de inmediato(21).
En contraste la guía de buenas prácticas para la seguridad del paciente en la atención en salud en Colombia, solo hace hincapié en un doble mecanismo de identificación que incluye el nombre del paciente y el número de cédula de ciudadanía el cual a su vez corresponde al número de historia clínica(17).

Según el Comité de Estándares de Calidad para Laboratorios Clínicos de Estados Unidos (NCCLS), existen muchas oportunidades de mejora continua de la calidad en esta fase en lo que respecta al procedimiento de identificación inequívoca de muestras o especímenes, ya que el objetivo final es garantizar que el tipo de muestra adecuado, se recolecte en el recipiente adecuado, en el momento correcto y a partir del paciente correcto. La identificación precisa del paciente es, por lo tanto, fundamental y no puede darse por sentado(22). Es por ello que el NCCLS y otros expertos internacionales han descrito que el rótulo de una muestra para estudio de gases en sangre debería contener como mínimo: nombre completo del paciente, edad/fecha de nacimiento, número de historia clínica, sala o servicio de hospitalización, cama de hospitalización, fecha y hora de toma de muestra, médico tratante, temperatura del paciente al momento de la toma de la muestra, estado de ventilación, sitio de toma de muestra, posición del paciente al momento de la toma de la muestra, tipo de anticoagulante usado, tipo de jeringa usada y persona que toma la muestra(23-25).

En este estudio se excluyeron cinco de estas variables (fecha de nacimiento, médico tratante, estado de ventilación, sitio de toma de muestra y posición del paciente al momento de toma demuestra) y de las recolectadas se cumplió, en el total de los casos, con el registro del número de historia clínica y la hora de toma de muestra. Luego, no se observó datos suficientes para la identificación inequívoca del paciente y en un alto porcentaje tampoco la identificación de factores que pudieran afectar la calidad. Al respecto en este último punto el paciente crítico no maneja una temperatura corporal normal y, debido a su enfermedad o tratamiento, son hipotérmicos o hipertérmicos. La hipotermia se asocia con una 
disminución de $\mathrm{PCO}_{2}$ y $\mathrm{PO}_{2}$, como consecuencia del aumento de la solubilidad de estos gases, y la hipertermia se asocia con un aumento de $\mathrm{PCO}_{2} \mathrm{y}$ $\mathrm{PO}_{2}$, como consecuencia de la disminución de la solubilidad(10). Adicionalmente, el cambio dependiente de la temperatura en la $\mathrm{PCO}_{2}$ afecta de manera secundaria al $\mathrm{pH}$ de la sangre; de tal forma que la hipotermia se asocia con un aumento del $\mathrm{pH}$ y la hipertermia con un descenso de este(11). En tal circunstancia, es apropiado aprovechar los algoritmos que comúnmente se proporcionan en el software de los analizadores de gases en sangre que permite que las mediciones realizadas a $37^{\circ} \mathrm{C}$ en el equipo, se corrijan matemáticamente a la temperatura corporal real del paciente. Este proceso no se llevó a cabo en más el $50 \%$ de las muestras incluidas en este estudio por la ausencia del dato de la temperatura del paciente.

Las variables $\mathrm{T}^{\circ} \mathrm{PT}$ y $\mathrm{TPT}$, están relacionadas. Basados en estudios experimentales limitados, se ha logrado establecer que la demora en el análisis puede disminuir la $\mathrm{PO}_{2}$ y aumentar la $\mathrm{PCO}_{2}$ en sangre arterial almacenada a $4^{\circ} \mathrm{C}$ debido al metabolismo celular(10). Sin embargo, la revisión de Baird,(11), describió que el exceso de exposición de las muestras en algunas jeringas plásticas a temperaturas $\leq 4^{\circ} \mathrm{C}$ trae problemas, primero porque en las muestras se duplica el coeficiente de solubilidad del oxígeno en la sangre (de 21,4 $\mathrm{mL} \mathrm{O}_{2} / \mathrm{L}$ de plasma a $37^{\circ} \mathrm{C}$ a $39,5 \mathrm{~mL} \mathrm{O}_{2} / \mathrm{L}$ de plasma a $4^{\circ} \mathrm{C}$ ) y segundo por el aumento de la afinidad entre el oxígeno y la hemoglobina, conduciendo a una disminución inicial de la $\mathrm{PO}_{2}$ en la muestra. Este fenómeno aumentaría el gradiente de oxígeno de la atmósfera a la muestra por la contracción del polímero, que conlleva a un ensanchamiento de los poros del material, los cuales parecen permitir el intercambio de moléculas de oxígeno con el medio externo, pero limitan el paso de las moléculas de dióxido de carbono mucho más grandes. Este comportamiento fue observado por Mohammadhoseini et al.(13.

Si bien analizar los cambios en la $\mathrm{PO}_{2}$ y $\mathrm{PCO}_{2}$ cuando se usan jeringas plásticas y almacenan muestras a diferentes temperaturas no fue objeto de estudio, es un trabajo interesante a considerar pues permitiría evaluar el impacto del tiempo y la temperatura en los actuales equipos de extracción(11,26,27).

Un porcentaje significativamente alto de las muestras de este estudio fueron trasladadas al laboratorio directamente por el personal de enfermería, en ellas el TPT fue inferior a los 30 minutos. Esto es lo ideal cuando se toman muestras para gases sanguíneos con jeringas plásticas(28,29), para Lippi et al.(2), así como para Delost(10) y Baird(11), no hay razón para mantener la muestra en agua helada si es procesada dentro de los primeros 30 minutos de tomada.

Por otra parte, no fue posible identificar el tipo de heparina usada tanto en las jeringas ya heparinizadas como en las que no tenían heparina, situación bajo la cual también debía considerarse la cantidad de heparina. Hay varios estudios que muestran la influencia del tipo de anticoagulante en la calidad de los resultados de gasometría sanguínea. El efecto interferente del anticoagulante sobre la muestra de sangre puede producirse por quelación o por dilución, si se usa un preparado líquido añadido en exceso en jeringas corrientes no liofilizadas con heparina $(30,31)$. Una cantidad inadecuada de anticoagulante puede afectar hasta en un $28 \%$ la $\mathrm{PCO}_{2}{ }^{(9)}$.

La presencia de burbujas de aire en las muestras también afecta la calidad de los resultados de la $\mathrm{PO}_{2}$ y pH. Calaf y Giner(32), llevaron a cabo un estudio para valorar la calidad de cinco equipos de extracción de sangre arterial, los resultados permitieron concluir que dos de los cinco equipos valorados permitían obtener muestras con menos burbujas y mayor facilidad para eliminarlas en el $44 \%$ de los casos. Esta variable fue considerada en este estudio, puesto que se evidenció la práctica de solicitar las jeringas herparinizadas a los familiares de los pacientes hospitalizados en las unidades fuentes de información, sin embargo, no fue posible recolectar el dato. Esto representa una debilidad en el estudio y una oportunidad para considerar en investigaciones futuras. LimaOliveira et al.,(33), así como Calaf y Giner(32), evaluaron cuatro tipos de jeringas, concluyendo que existían diferencias significativas entre los 
resultados del pH según equipo de extracción usado, los cuales iban acompañados de una disminución de la $\mathrm{PCO}_{2}$, debidas probablemente a la fuga de gas a través del material plástico de los dispositivos de recolección, además, uno de los equipos contaminaba menos la muestra.

Muchas pueden ser las causas de los errores preanalíticos en los estudios de gasometría, incluyen tanto factores del paciente debidos a la presencia de interferencias por fármacos o dietas especiales, como por el procedimiento de toma de muestra y manejo pre-analítico(22,23). Sin embargo, para Baird(11) y Youssef et al.(34), es posible hacer una buena mitigación de estos factores con procesos de capacitación continua, sensibilización de la importancia del manejo urgente o prioritario de estas muestras así como la estandarización de los procedimientos y evaluación de la adherencia a estos, a través del diseño de listas de chequeo para el control de calidad de las muestras que ingresan al laboratorio, del proceso de toma de muestras y gestión de la misma en la fase pre-analítica, entre otros. Sin embargo, son estrategias que en su conjunto nunca proporcionan el $100 \%$ de efectividad de aquí la importancia de la gestión del riesgo en la fase pre-analítica, que la mayoría de las veces se encuentra fuera del control del personal del laboratorio clínico debidamente entrenado y capacitado(35).

\section{Conclusión}

Se identificó un bajo cumplimento de los estándares técnicos de las variables de temperatura y tiempos de traslados para gasometría. Estas dos son variables relacionadas, de manera que un efecto indeseable en una, impacta directamente en la otra. De aquí la importancia de controlar simultáneamente las dos variables. Se evidenció un bajo cumplimiento global de la guía nacional para la identificación de pacientes, solicitudes y muestras del Ministerio de la Protección Social para la seguridad del paciente. Estos lineamientos son insuficientes para la gestión del riesgo y seguridad del paciente desde el laboratorio clínico. La fase pre-analítica es una fase muy manual, razón por la cual, el primer paso para mejorarla es la documentación, estandarización y normalización de sus procedimientos que posteriormente han de ser socializados con el personal y controlados a través de listas de chequeo que permitan consolidar datos para el diseño y cálculo de indicadores de control. La responsabilidad de la calidad de los resultados analíticos es del personal de laboratorio y si bien, la de toma de muestra no es llevada por este, es imprescindible que los expertos del análisis clínico aporten su conocimiento y experticia en el diseño y vigilancia del cumplimento de los procedimientos preanalíticos extralaboratorio que afectan sus mediciones.

\section{Limitaciones y fortalezas}

El no haber identificado el tipo de jeringa y el anticoagulante limitó mucho el análisis de los resultados, considerando estas fallas técnicas en el equipo de extracción. Los estudios identificados en la revisión bibliográfica indicaban la pertinencia de su valoración por institución con el fin de definir parámetros objetivos de compra de estos insumos. Por otra parte, no se recolectó la variable cantidad de volumen de muestra y la presencia de burbujas de aire, factores determinantes en la $\mathrm{PO} 2, \mathrm{PCO}_{2}$ y $\mathrm{pH}$.

\section{Fuentes de financiación}

Este trabajo fue financiado por la Vicerrectoría de Investigaciones de la Universidad del Valle, bajo el radicado 1721.

\section{Agradecimientos}

Los autores agradecen a la institución fuente de información.

\section{Conflicto de intereses}

No existen conflictos de interés.

\section{Referencias}

1. Sánchez-Alarcos JM, Álvarez-Sala JL. Interpretación de la gasometría arterial. Medicine [Internet]. 2006; 9(68):4406-4409. Available from: https://www.sciencedirect.com/sdfe/pdf/download/ei d/1-s2.0-S0211344906744459/first-page-pdf.

2. Lippi G, Mattiuzzi C, Bovo Ch. Are we getting better at the preanalytical phase or just better at measuring it?. J Lab Precis Med [Internet]. 2018;3:11. Available from: http://jlpm.amegroups.com/article/view/3992/4746 
3. van Dongen-Lases EC, Cornes MP, Grankvist K, Ibarz M, Kristensen GB, Lippi G, Nybo M, Simundic AM; Working Group for Preanalytical Phase (WG-PRE), European Federation of Clinical Chemistry and Laboratory Medicine (EFLM). Patient identification and tube labelling - a call for harmonisation. Clin Chem Lab Med [Internet]. 2016;54(7):1141-5. Available from:https://www.degruyter.com/downloadpdf/j/ccl m.2016.54.issue-7/cclm-2015-1089/cclm-20151089.pdf.

4. Sintes N. Errores pre-analíticos en la extracción de una muestra para gasometría arterial. Revisión sistemática. Revista ASDEC [Internet]. 2018:(10):0. Available from: http://cursosfnn.com/articulo/errores-pre-analiticosen-la-extraccion-de-una-muestra-para-gasometriaarterial-revision-sistematica/\#resultados-y-discusion.

5. Chhapola V, Kumar SA, Goyal P. Is liquid heparin comparable to dry balanced heparin for blood gas sampling in intensive care unit?. Indian Journal of Critical Care Medicine [Internet]. 2014;18(1):14-20. Available

from:http://www.ijccm.org/temp/IJCCM181143459503_093635.pdf.

6. Sareen R, Kapil M, Gupta GN. Preanalytical variables: Influence on laboratory results and patient care. Int J Clinicopathol Correl [Internet]. 2017;1:31-4. Available from: http://www.ijcpc.org/article.asp?issn=WKMP0139; year $=2017$; volume $=1$; issue $=1 ;$ spage $=31$; epage $=3$ 4 ;aulast=Sareen.

7. Mohammed H, Abdallah D. Easy blood gas analysis: Implications for nursing. Egyptian Journal of Chest Diseases and Tuberculosis [Internet]. 2016;65:369-376. Available from: https://core.ac.uk/download/pdf/82018845.pdf.

8. Englezopoulou A, Kechagia M, Chatzikiriakou R, Kanellopoulou M, Valenti M and Masedu F. Pre Analytical Errors as Quality Indicators in Clinical Laboratory. Austin J Public Health Epidemiol [Internet]. 2016;3(5):8. Available

from: file:///C:/Users/Usuario/Downloads/fulltext_ajphev3-id1048\%20(1).pdf.

9. Kumar A, Kushwah S, Sahay Sh. Effect of extra amount of heparin in syringe and its effect on arterial blood gas analysis. EJPMR [Internet]. 2015;2(6):290-293. Available

from:http://www.ejpmr.com/admin/assets/article_iss ue/1447552403.pdf.

10. Delost M. Blood gas and critical care analyte analysis. Chapter 6. [Internet]. Editorial Jones \& Bartlett Learning. 2014. Available from: http://samples.jbpub.com/9781449652838/Chapter6. pdf.

11. Baird G. Preanalytical considerations in blood gas analysis. Biochem Med (Zagreb) [Internet]. 2013;23(1):19-27. Available from:http://www.biochemiamedica.com/assets/images/upload/xml_tif/Baird_G_Preanalytical_considerations_in_blood_gas_analysis.pdf.

12. Dukić L, Milevoj L, Dorotić A, Baršić I. Blood gas testing and related measurements: National recommendations on behalf of the Croatian Society of Medical
Biochemistry and Laboratory Medicine. Biochem Med (Zagreb) [Internet]. 2016;26(3):318-336. Available from:

https://www.ncbi.nlm.nih.gov/pmc/articles/PMC5082 214/.

13. Mohammadhoseini E, Safavi E, Seifi S, Seifirad S, Firoozbakhsh S, Peiman S. Effect of Sample Storage Temperature and Time Delay on Blood Gases, Bicarbonate and pH in Human Arterial Blood Samples. Iranian Red Crescent medical journal [Internet]. 2015;17(3):e13577.Available from: https://www.ncbi.nlm.nih.gov/pmc/articles/PMC4441 $774 /$.

14. Srisan P, Udomsri T, Jetanachai $\mathrm{P}$, Lochindarat $\mathrm{S}$, Kanjanapattanakul W. Effects of temperature and time delay on arterial blood gas and electrolyte measurements. J Med Assoc Thai. 2011;94(3 Suppl):S914.

15. Hedberg P, Majava A, Kiviluoma K, Ohtonen P. Potential prenalytical errors in whole-blood analysis: effect of syringe sample volume on blood gas, electrolyte and lactate values. Scand J Clin Lab Invest [Internet]. 2009;69:585-591. Available from: https://www.tandfonline.com/doi/abs/10.1080/0036 5510902878716?journalCode=iclb20.

16. García-García JA, Reding-Bernal A, López-Alvarenga JC. Cálculo del tamaño de la muestra en investigación en educación médica. Inv Ed Med [Internet]. 2013;2(8):217-224. Available from: http://riem.facmed.unam.mx/sites/all/archivos/V2Nu m04/07_MIE_CALCULO_DEL_TAMANO.PDF.

17. República de Colombia. Ministerio de Salud. Guía de buenas prácticas para la seguridad del paciente en la atención en salud. Paquetes instruccionalespara la correcta identificación del paciente y de las muestras en el laboratorio clínico. Versión 2. Available from: https://www.minsalud.gov.co/sites/rid/Lists/Bibliotec aDigital/RIDE/DE/CA/Guia-buenas-practicasseguridad-paciente.pdf.

18. Grupo de Trabajo de Seguridad del Paciente. Sociedad Española de Bioquímica y Patología Molecular. Estudio de la oxigenación e interpretación de la gasometría arterial. Documentos de la SEQC [Internet]. 2015:31-47. Available from: http://www.seqc.es/es/Comisiones/18/61/Grupo_de_ Trabajo_sobre_Seguridad_del_paciente_\%7C_Comite_Ci entifico/.

19. Sánchez VM, Muñoz MR, Chávez C, Guerrero I. Utilidad de los parámetros gasométricos como predictores de mortalidad en pacientes con choque séptico. Med Crit [Internet]. 2017;31(3):128-13. Available from: http://www.medigraphic.com/pdfs/medcri/ti2017/ti173d.pdf

20. Hawkins R. Managing the pre- and post-analytical phases of the total testing process. Lab Med [Internet].2012;32:5-1. Available from: https://www.ncbi.nlm.nih.gov/pmc/articles/PMC3255 $486 /$.

21. Lippi G, Chiozza L, Mattiuzzi C, Plebani M. Patient and Sample Identification. Out of the Maze?. Journal of medical biochemistry [Internet]. 2017;36(2):107-112. 
Available

from:

https://www.ncbi.nlm.nih.gov/pmc/articles/PMC5471 $642 /$.

22. National Committee for Clinical Laboratory Standards. NCCLS. Procedures for the Collection of Arterial Blood Specimens; Approved Standard-Fourth Edition. NCCLS document, H11-A4. Wayne, PA, USA: NCLSI; 2004.

23. Plebani $M$. The detection and prevention of errors in laboratory medicine. Ann Clin Biochem. 2010;47:10110.

24. Mohammedsaleh ZM, Mohammedsaleh F. A Review Article of the Reduce Errors in Medical Laboratories. Glob J Health Sci [Internet]. 2015;7(1):46-51. Available from:

https://www.ncbi.nlm.nih.gov/pmc/articles/PMC4796 $466 /$.

25. Lippi G, Plebani M. Blood tubes should be labeled before drawing blood. Ann Blood [Internet]. 2017;2:18. Available from: http://aob.amegroups.com/article/view/4171/4901.

26. Mahoney JJ, James A. Harvey, Wong RJ, Van Kessel AL. Changes in oxygen measurement when whole blood is stored in iced plastic syringe or glass syringes. Clin Chem. 1991;37(7):1244-48.

27. Knowles TP, Mullin RA, Hunter JA, Douce FH. Effects of Syringe Material, Sample Storage Time, and Temperature on Blood Gases and Oxygen Saturation in Arterialized Human Blood Samples. RESPIRATORY CARE [Internet]. 2006;51(7):732-736. Available from: http://rc.rcjournal.com/content/51/7/732/tab-pdf

28. Smajić J, Kadić D, Hasić S, Serdarević N. Effects of postsampling analysis time, type of blood samples and collection tubes on values of blood gas testing. Med Glas (Zenica) [Internet]. 2015; 12(2):1-5. Available from: http://ljkzedo.ba/sites/default/files/unovombroju/13 \%20Smajic\%20823\%20A.pdf.

29. Wagner D. The physiological basis of pulmonary gas exchange: implications for clinical interpretation of arterial blood gases. Eur Respir J [Internet]. 2015;45:227-243. Available from: http://erj.ersjournals.com/content/45/1/227.long.

30. Chhapola V, Kumar SA, Goyal P. Is liquid heparin comparable to dry balanced heparin for blood gas sampling in intensive care unit?. Indian Journal of Critical Care Medicine [Internet]. 2014;18(1):14-20. Available from: http://www.ijccm.org/temp/IJCCM181143459503_093635.pdf.

31. Kumar A, Kushwah S, Sahay Sh. Effect of extra amount of heparin in syringe and its effect on arterial blood gas analysis. EJPMR [Internet]. 2015;2(6):290-293. (Available

from: http://www.ejpmr.com/admin/assets/article_issue/14 47552403.pdf.

32. Calaf N;Giner J. Comparison of arterial blood sample kits. Arch Bronconeumol. Arch Bronconeumol [Internet]. 2004;40(8):377-80. Available from: http://www.archbronconeumol.org/en-pdfS1579212906603248.

33. Lima-Oliveira G, Lippi G, Salvagno GL, Picheth MG,Guidi GC. Different manufacturers ofsyringes: A new source of variability in blood gas, acidâ base balance and related laboratory test?. Clinical Biochemistry. 2012;45(9):683687.

34. Youssef W, Yahia A,Shaaban N, Elhabashy S. Factors Affecting Validity of Arterial Blood Gases Results among Critically Ill Patients: Nursing Perspectives. Journal of Education and Practice. 2013;4(15):43-56.

35. Figueroa-Montes LE. Gestión de riesgos en los laboratorios clínicos. Acta Med Per [Internet].. 2015;32(4):241-250. Available from: http://www.scielo.org.pe/pdf/amp/v32n4/a08v32n4. pdf 